\title{
Face Recognition Outside the Visible Spectrum
}

\author{
Thirimachos Bourlai and Lawrence A. Hornak
}

\begin{abstract}
Automated face recognition (FR) is a well-studied problem, with a history of more than three decades. Facial recognition software that uses two dimensional (2D) images has advanced significantly in terms of accuracy over recent years. Despite the steps forward, face recognition is still not considered to be a solved problem for the cases of (i) difficult exposure conditions, such as during night-time, or in environments with unconstrained lighting, (ii) when operating at long standoff distances or at variable distances from the camera, and (iii) when using different camera sensors that operate in the same or different spectral bands. In practical forensic scenarios, it is often the case that investigators need to operate in difficult conditions, where face images captured in the aforementioned challenging cases need to be matched against good quality face images (gallery set) and where, grouping of the data in the context of demographic information (in terms of gender, ethnicity or other soft biometrics) may also be used in order to assist law enforcement officials, forensic investigators and security personnel in subject identification. In fact, in such practical scenarios, human recognition based solely on visible spectral images may not be feasible. Hence, an attractive solution is to develop efficient FR systems that can work in different practical scenarios. While different systems have been discussed and proposed over the years, an interesting approach is to increase the plurality of the source data, or in other words, start using data captured by camera sensors that cover a broad range of the electromagnetic spectrum that falls outside the visible band, including the ultraviolet $(U V)$ and the infrared (IR) band. Thus, FR outside the visible spectrum is considered an area of growing research interest. This is further supported by the burgeoning market of camera sensors that continues to open new opportunities, at different spectral and spatial scales, in a large number of applications including those in security, forensics and defense. In light of these thoughts, the focus of this work is to expose readers to a plethora of FR advancements when operating outside the visible spectrum and our perspective on where this field is going. The discussion will include the latest studies in the UV (100-400 nm), active IR band (0.7-2.5 $\mu \mathrm{m})$ and, finally, the passive IR band, or more specifically, the mid-wave IR (3-5 $\mu \mathrm{m})$ and long-wave IR (8-14 $\mu \mathrm{m})$ bands. The discussion will also include research work that involves same-spectral and cross-spectral matching scenarios, where, first, face images (probes) are acquired under controlled or difficult conditions as explained above, including dealing with uncooperative subjects, and adverse environments. Then, depending on the FR scenario, the acquired probe images are matched against mug shots collected in a controlled indoors environment using a conventional camera. This paper will start by briefly covering different multi-spectral FR systems and, then, discussing various related topics (ranging from data acquisition sets, up to system performance). The paper will also suggest needed new avenues of inquiry in the context of current work in multi-spectral FR system.
\end{abstract}

Keywords Face Recognition · Ultraviolet Imagery · Visible Imagery · Near Infrared Imagery · Short-Wave Infrared Imagery · Mid-Wave Infrared Imagery · Long-Wave Infrared Imagery · Cross-Scenario Face Matching · Night-time Imaging · Long-Distance Imaging

Thirimachos Bourlai, Assistant Professor

West Virginia University: Benjamin M. Statler College of Engineering and Mineral Resources, PO Box 6070, Morgantown, WV 26506 Tel.: +1-304-293-4326; Email: ThBourlai@mail.wvu.edu

Lawrence A. Hornak, Professor and Associate Dean for Research

University of Georgia: College of Engineering, 130 Paul D. Coverdell Center, Athens, GA 30602

Tel.: +1-706-542-2462; Email: lahornak@uga.edu 


\section{Introduction}

Biometric systems utilize physiological (face, fingerprints, iris etc.) or behavioral (keystroke dynamics etc.) traits to establish human identity. Face-based recognition (FR) systems, in particular, are gaining interest because the human face has several advantages over other biometric traits. For example, it is nonintrusive, understandable, and can be captured at variable standoff distances. A typical FR system includes an enrollment phase, during which images of the users face are taken and used to create a template that is stored in a database (gallery images). During the authentication phase, newly recorded images of a users face, called probes, are used for recognition. A decision on the persons identity is made on the basis of the comparison between gallery templates and new (probe) images. Most of the well-studied FR systems depend on the use of face images captured in the visible range of the electromagnetic spectrum, i.e. 380-750 $\mathrm{nm}$. Hence, the field of visible-based FR using 2D images has advanced significantly. There are various important studies reported, such as [3,4] from the NIST Information Technology Laboratory (ITL) that has developed standardized test methods, providing a reliable way to evaluate the accuracy of different FR algorithms against known datasets of images (one-to-many). In these visible range studies, the evaluations mainly focused on posed images, including mugshots, visas, passport photos, and drivers licenses, and demonstrated that the accuracy of the algorithms has increased as much as 30\% in recent years [4].

But what happens when we move away from the visible band and we have to work in difficult environments? In military and law enforcement situations, FR system operators have to deal with challenging conditions characterized by unfavorable lighting and pronounced shadows. Such an example is a night-time environment, where human recognition based solely on visible spectral images may not be feasible [1], [2]. In practice, the potential for imaging outside the visible spectrum to enhance facial recognition, may present itself in a variety of ways that are complementary to computer vision and pattern recognition methods applied when using face data from standard color or monochrome cameras. One complementary way is to use non-visible spectral imaging to reliably detect human skin and segment the face from the background environment for further processing. An alternative way is to use non-visible spectral imaging so that spatial inhomogeneities can be enhanced, i.e. inhomogeneities that are not clearly visible to the naked-eye in the visible band or easily exploited by conventional algorithmic approaches. Such an example is the vascularrelated patterns, which can be unique to each person.

Thus, while in recent years the FR community has grown immensely, from our perspective, there are some things lacking that would help make the FR field even more successful. Our opinions are based on our association with the subject and also our experience in different but related disciplines (including computer vision, medical image processing, machine learning, optical science and engineering, and other efforts in the field of entrepreneurship and innovation). The views expressed in this article are influenced by various discussions at different academic but also non-academic focused conferences and workshops, which bring together government and industry experts at the forefront of developing the next generation of biometrics.

\subsection{A Potential Step Forward}

One of the suggested avenues that FR research can move towards is to increase the focus in the usage of multi-spectral and hyper-spectral camera sensors that will help the community better deal with difficult FR scenarios. In fact, such a usage, whenever possible (cost and scenario dependent), will have a better impact if it is combined with state-of-the-art pre-processing, feature extraction and matching algorithms. The proposed avenue is expected to increase the probability of accurate recognition, because while spatial information of the face can be exploited (e.g. texture and topology), spectral-specific facial information can be exploited in parallel, taking advantage of the fact that the spectral-specific signature of the face of each individual can be unique. The viability of multi-spectral image acquisition continues to improve, with the range of technologies, their quality and cost improving significantly. Focal plane array devices are available for the UV-Visible, near infrared (IR), Short-wave IR (SWIR), Mid-wave IR (MWIR) and Long Wave IR (LWIR). While the economy of these technologies continues to vary significantly, downward pressure continues on cost as technologies mature, both pixel densities and good pixel yield increases, and applications expand.

But what is the basis of the claim that spectral imaging can be important in biometrics when combined with spatial information? Spectral imaging of human tissue at a given electromagnetic wavelength is based on a specific combination of material interactions, including those with water, melanin, and hemoglobin 
[5]. This combination results in humans having a distinct facial skin from a variety of backgrounds, mainly because of the inhomogeneous distribution of the aforementioned components that varies across the skin of different individuals. As a result, when working with face images covering different spectral bands or narrow wavelengths, the spatial component of these images provides a basis for making spectral distinctions of these differences. Thus, spectral and spatial information is thought to provide an improved facial-based signature that with proper choice of spectrum, can be less affected by variations in facial expression, make-up or aging. The question becomes "what portion of the spectrum, or which specific bands need to be further investigated to achieve better FR performance results on challenging scenarios?". There are various studies in the literature that offer encouraging results, which further support the need to accelerate truly interdisciplinary research in multi-spectral and hyper-spectral imaging for FR applications that brings together optical science and engineering with computer science for hardware and algorithm co-design. The next section briefly discusses the advantages of spectral imaging, including new findings in the field of homogeneous and heterogeneous FR, when operating at different spectral bands and/or wavelengths.

Perhaps when working with multi-spectral FR what is needed is: (i) The development of advanced computer vision (CV) algorithms with improved face/eye detection and FR capabilities, including single or multi-channel convolutional neural networks (CNNs), image synthesis (for low cost transition from one to another spectral band) etc. (ii) Better collaboration among academic institutions (including Institutional Review Boards) when face image and video data are collected and, then, are needed to be shared among researches to conduct studies. This is because the data collection cost can be significant, especially when (a) having to use many different imaging sensors, where the cost of some of those may be ranging from several $\$ 10 \mathrm{~K}$ to more than $\$ 150 \mathrm{~K}$ (e.g. specialized thermal imaging sensors), (b) having to recruit and reimburse from hundreds to thousands or even millions of subjects (e.g. 600 million citizens of India were enrolled with biometric ID by about 2014 - J. Daugman, 2014, SPIE Newsroom). By better designing and executing coordinated data acquisition and sharing efforts the field will advance much faster. (iii) Lastly, further collaboration between computer scientists and physical/optical scientists in order to understand the origin, uniqueness and permanence of multi-spectral biometric-related features.

\section{Advantages and Limitations of Spectral Imaging: Towards Face Recognition}

While facial recognition technology has matured to be used as a reliable and routinely applied tool, there is much room for improvement. Some factors that are reducing performance are sensitivity to variable illumination geometries, changes in facial expression, and nighttime conditions. Spectral imaging is one of several modalities (e.g., 3D imaging) that may enhance the performance of facial recognition systems. Spectral imaging can provide additional information both within and outside of the visible spectrum, including the ultraviolet and infrared. What follows is a brief discussion of these bands.

The UV band is positioned within the electromagnetic spectrum between the X-ray and the VIS band. The UV band ranges from 100-400 nm, and consists of the three bands: the long wave UV-A band (315-400 $\mathrm{nm})$, the medium UV-B (280-315 nm) and the short wave UV-C band (100-280 nm). UV-A light sources, such as metal-halide flash lamps, are used in flash photography at power levels which are eye-safe and adequate for imaging. In the case of the UV face images, there are significant differences in appearance when compared with visible ones. Due to the fact that UV and VIS bands are close, and both UV and visible face images can be captured using high quality camera sensors, the study of FR using data from those bands is a promising area of research. UV imagery can generate a more complete face representation, where various interesting facial features, including skin texture are revealed. Therefore, the UV band may take advantage of the facial features that might go unobserved. There are various groups working with UV images. Cooksey et al. [6] performed the reflectance measurements of the human skin ranging from the UV to the IR band. Burgener et al. [8] proposed the risks and benefits associated with the exposure to the UV band in biological cabinets. In [9] the authors performed UV vs. UV and UV vs. VIS FR experiments. In the latter scenario they achieved up to $98 \%$ rank-1 identification rate even when using standard academic algorithms.

While active IR bands depend critically on the illuminating light source and the reflective and scattering behavior of the subject, IR active imaging systems that operate at these bands can provide complementary or finer facial details than found in standard RGB visible imagery. The active IR band can be divided into the NIR (750-1100 nm) and SWIR (900-1900 nm) bands [10][11][12]. NIR and SWIR bands promote a more 
natural transition from visible-based mug shot galleries for both same- and cross-spectral FR. But what are the pros and cons when operating in the active IR band? In low light situations, active IR imagery has a distinct advantage over visible imaging, namely the ability to capture face images in low-light scenarios (dusk and twilight) and poor environmental conditions (fog, haze, or particulate in the air sufficient to affect image quality). In addition, at certain wavelengths, reflective coated glass and tinted materials can become transparent [13], while certain facial features can become more prominent with additive active-illumination.

In no-light environments, eye-safe active illumination can be applied safely and covertly if/when necessary. In law enforcement applications active IR imaging systems are widely used. However, there are many silicon image sensors that can detect NIR active illumination sources when used. In fact, there are situation where the human eye can also detect NIR illumination. In terms of the SWIR band, it offers eye-safe, notdetectable illumination above $1400 \mathrm{~nm}$, while it is also invisible to silicon-based image sensors [15]. SWIR images (probes captured in night-time and low-light conditions) can be efficiently matched against visible band face images with surprisingly high scores. One of the drawbacks of SWIR imaging is that face images captured above $1450 \mathrm{~nm}$ are significantly affected and appear dark (due to absorption by the moisture found in human skin above this wavelength) [16]. This problem intensifies when eye-safe illumination is used. As the wavelength increases, the darkening effect of mucous membranes, such as the eye, becomes more prominent. This poses a major challenge to detection algorithms that focus on eye tracking. The entire eye may appear black, making the pupil, iris and sclera indistinguishable. Skin oil may also create matching difficulties in SWIR due to strong reflections and resulting camera saturation.

When moving to the passive IR band, the camera sensor detects IR radiation in the form of heat, which is emitted from the subjects face. As discussed, it is divided into the MWIR and LWIR band. Recent FR research illustrated that working in the passive IR band is very challenging. However, thermal-based camera sensors can be used for difficult FR scenarios such as low light or complete dark environments. When subjects are within an appropriate distance from the passive camera we can achieve obscure detection and acquisition of face images. The appearance of MWIR and LWIR face images look visually similar, but they both capture different facial characteristics. MWIR has both reflective and emissive properties and works better for long-range operations. In contrast, LWIR consists primarily of the subjects emitted radiation. Some other advantages are: (i) the fact that in either band, face images are not normally affected by external illumination, (ii) unique physiological facial features for individuals (e.g. veins, edges, and wrinkles), are detectable in this band, and thus, useful for human recognition. Finally, image acquisition in these passive bands is unobtrusive, and therefore passive IR FR is useful for both military and law enforcement applications. The authors in [16] reported that FR in the LWIR band achieves a rank-1 accuracy of $97.3 \%$ when using local binary patterns (LBP), while no cropping or geometrical normalization step is required. In Socolinsky et al. [1], the authors used two standard FR algorithms to show that, under variable illumination conditions, the usage of LWIR face images yield a higher recognition performance than visible ones. However, the drawback of the approach is that LWIR and visible images were divided into multiple training and testing sets resulting in an increase of the FR system design time. In addition, the study was performed using co-registered images that were captured simultaneously by both visible and LWIR cameras; and this is not usually possible in operational environments. In other IR-based FR approaches such as Trujillos et al. [17], the authors proposed an unsupervised local and global feature extraction paradigm to classify different facial expressions. In Chen et al. [18] the authors combined visible and MWIR images and compared them using Principle Component Analysis. Neither Trujillo et at. nor Chen et al. focused in MWIR FR. Finally, in [19] a unified face matching algorithm is reported that works efficiently in the visible and passive IR bands.

\section{Concluding Remarks: Moving Forward}

It is clear there is strong potential in multi-spectral imaging. While there are still high-end military systems that can be too expensive for academia, camera costs are reducing and spectral bands are becoming available as focal plane device technology improves, driven by a broader application pull. While great progress has been made, the target is a rapidly moving one and the community needs to organize to advance the field similar to what it did for visible band FR. Moving forward, to effectively organize and advance multi-spectral FR, there is strong need for increased collaboration between the medical scientists, optical scientists and engineers, and computer scientists. This is necessary for various reasons. First, the community 
need for publicly accessible, well-designed data sets that span the available multi-spectral ranges and are of reasonable size. These datasets need to be acquired not only by using best practices but also draw on interdisciplinary input in their design. Given the cost and complexity of such collections, which may use a range of multi-spectral equipment, interdisciplinary input will be essential in designing collections that reveal salient information regarding multi-spectral features and are most useful for algorithm design and development. Secondly, this interdisciplinary team approach is essential given the expanded ability multi-spectral imaging provides to effectively acquire an entirely new set of features from the human body. Collaborative work continues to be needed to fully understand these surface and subcutaneous human features, their physiological origin and their optical characteristics across different spectral bands. Only with complete information can feature uniqueness and permanence be properly assessed and, together with other considerations (such as application dependent operational constraints), can useful system design decisions be made. Thirdly, we need to continue the development of advanced CV algorithms with improved detection and matching capabilities, including single or multi-channel CNNs, image synthesis etc. Finally, we need to be thinking how we can better transition technology from academia to business. What is needed is a better standardization of system evaluation processes, including continuous monitoring of performance of baseline systems when scenarios change; the management and development of more efficient and effective large-scale operational capabilities and the establishment of standards for plug-and-play performance and system interoperability; more sharing of biometrics recognition code; and more dialogue with the customer and the government.

Acknowledgements This material is sponsored partly through the WVU start-up funds of Prof. T. Bourlai, the Center for Identification Technology Research and the National Science Foundation under Grant No. - West Virginia University 1066197, and finally, through a Grant from the Office of Naval Research (N00014-08-1-0895), "Distribution A-Approved for Unlimited Distribution". We are also grateful to WVU faculty, staff and students for all their help and support.

\section{References}

1. Selinger A. and Socolinsky D. A.: Face Recognition in the Dark, In Proc. Conference on Computer Vision and Pattern Recognition Workshop, pp. 129134, Washington, DC, USA, June (2004).

2. Ao M., Yi D., Lei Z., and Li S. Z.: Handbook of Remote Biometrics for Surveillance and Security. Advances in Computer Vision and Pattern Recognition Series, Springer, (2009).

3. Grother, P. J., Quinn, G. W. and Phillips, P. J.: Report on the Evaluation of 2D Still-Image FR Algorithms. NIST Inter-agency, Internal Report (NISTIR) - 7709, June (2010).

4. Grother, P. J., and Ngan, M. L.: Performance of Face Identification Algorithms. NIST Inter-agency, Internal Report-8009, (2014).

5. David W. Allen (NIST): Chapter 1. An Overview of Spectral Imaging of Human Skin toward Face Recognition. In: T. Bourlai (Ed.), Face Recognition Across the Imaging Spectrum. London, U.K., Springer London Ltd., (2016).

6. Cooksey, C. C. and Allen, D. W.: Reflectance Measurements of Human Skin from the Ultraviolet to the Shortwave Infrared (250 $\mathrm{nm}$ to $2500 \mathrm{~nm}$ ). In: Proceedings of the SPIE Conference on Active and Passive Signatures (SPIE), vol. 8734 (2013).

7. Fulton, J. E.: Utilizing the Ultraviolet (UV detect) Camera to Enhance the Appearance of Photo-damage and other Skin Conditions. Dermatological Surgery, vol. 23, no. 3, pp. 163-169, (1997).

8. J. Burgener, J.: Position Paper on the Use of UV Lights in Biological Safety Cabinets. Appl Biosaf. vol. 11, pp. 228-230, (2006).

9. Narang, N., Bourlai, T. and Hornak L. A.: Can we Match UV Face Images Against their Visible Counterparts? , In: Proc. SPIE 9472, Algorithms and Technologies for Multi-spectral, Hyper-spectral, and Ultra-spectral Imagery XXI, 94721Q, (2015).

10. Kalka, N.D., Bourlai, T., Cukic, B., and Hornak, L.: Cross-spectral Face Recognition in Heterogeneous Environments: A Case Study on Matching Visible to SWIR imagery. In: Proc. of Int. Joint Conf. on Biometrics, pp. 1-8 (2011).

11. Zhu, J.Y., Zheng, W.S., Lai, J.H., and Li, S.Z.: Matching NIR face to Visible Face using Transduction. IEEE Transactions on Information Forensics and Security, 9(3), 501-514 (2014).

12. Klare, B., and Jain, A.K.: Heterogeneous FR: Matching NIR to Visible Light Images. In: Proc. of Int. Conf. on Pattern Recognition, pp. 1513-1516 (2010)

13. Nicolo, F., and Schmid, N.A.: Long Range Cross-spectral FR: Matching SWIR against Visible Light Images. IEEE Trans. on Inf. Forensics and Security 7(6), 1717-1726 (2012).

14. Lemoff, B.E., Martin, R.B., Sluch, M., Kafka, K.M., McCormick, W., Ice, R.: Long-range Night/Day Human Identification using Active-SWIR Imaging. In: SPIE Defense, Security, and Sensing, pp. 87,042J-87, 042J, (2013).

15. Chang, H., Yao, Y., Koschan, A., Abidi, B., Abidi, M.: Spectral Range Selection for FR under Various Illuminations. In: IEEE International Conference on Image Processing (ICIP), pp. 2756-2759, (2008)

16. Méndez, H., San Martín, C., Kittler, J., Plasencia, Y., García-Reyes, E.: FR with Long-wave IR Imagery using Local Binary Patterns. In: Massimo, T. and Nixon, M. S. (Ed.), Advances in Biometrics: Lecture Notes in Computer Science, vol. 5558, pp. 327-336. Springer Berlin Heidelberg (2009).

17. L. Trujillo, G. Olague, R. Hammoud, and B. Hernandez: Automatic Feature Localization in Thermal Images for Facial Expression Recognition. In: IEEE Computer Vision and Pattern Recognition, (CVPR), vol. 2, pp. 1-14, (2005).

18. X. Chen, P. Flynn, and K. Bowyer: PCA-Based Face Recognition in Infrared Imagery: Baseline and Comparative Studies. In: IEEE International Workshop on Analysis and Modeling of Faces and Gestures (AMFG), (2003).

19. Osia, N. and Bourlai, T.: A Spectral Independent Approach for Physiological and Geometric-based FR in the Visible, MWIR and LWIR Bands. In: Elsevier - Image and Vision Computing, vol. 32, no. 11, pp. 847-859, (2014). 